La

Révolution

française

\section{La Révolution française}

Cahiers de l'Institut d'histoire de la Révolution française

$20 \mid 2021$

La Révolution en 3D - Textes, images, sons

(1787-2440)

\title{
Claude Simon et la Révolution : écrire l'opacité et la désaffiliation
}

Claude Simon and the Revolution: writing opacity and disaffiliation

\section{Louis Hincker}

\section{(2) OpenEdition \\ Journals}

Édition électronique

URL : https://journals.openedition.org//rf/5105

DOI : $10.4000 /$ Irf.5105

ISSN : 2105-2557

Éditeur

IHMC - Institut d'histoire moderne et contemporaine (UMR 8066)

Référence électronique

Louis Hincker, «Claude Simon et la Révolution : écrire l'opacité et la désaffiliation », La Révolution française [En ligne], 20 | 2021, mis en ligne le 25 juin 2021, consulté le 28 juin 2021. URL : http:// journals.openedition.org//rf/5105; DOI : https://doi.org/10.4000/Irf.5105

Ce document a été généré automatiquement le 28 juin 2021.

(C) La Révolution française 


\title{
Claude Simon et la Révolution : écrire l'opacité et la désaffiliation
}

Claude Simon and the Revolution: writing opacity and disaffiliation

\author{
Louis Hincker
}

\section{NOTE DE L'ÉDITEUR}

Cet article est basé sur une communication présentée lors du colloque « La Révolution en 3D - Textes, images, sons (1787-2440) » qui s'est tenu à l'université Paris 1 PanthéonSorbonne du 14 au 16 mars 2019, organisé par le Cespra et l'IHMC-IHRF. Vous pouvez retrouver cette communication sur la chaîne YouTube de l'IHMC à l'adresse : https:// youtu.be/VhBKptGVTwY

1 Au tournant des années 2010, pas moins de sept revues françaises ont voulu de nouveau interroger la relation entre Histoire et Littérature, afin de se demander si cette dernière devait effectivement être considérée pour sa force supplétive, en tant qu'elle proposerait de combler des vides de la connaissance, de l'entendement ${ }^{1}$. Dans ce débat, que l'on refuse cette proposition ou que l'on accepte et assume que l'historien ne puisse effectivement avoir le dernier mot, il semble entendu que le droit de l'imagination, ou à l'imagination, veuille dire parer à une lacune, propice donc à dépasser des apories, gage de désinhibition. On peut craindre que, dans ces conditions, les textes de Claude Simon ne puissent y faire entendre leur voix, à moins de savoir goûter leur saveur d'inactualité. Ces textes appartiennent à une littérature pour laquelle il y a un lyrisme des espaces laissés en blanc sur la page, sans illusion sur l'insatiable voracité contemporaine visant des vérités non formulées. Un roman comme Les Géorgiques, qui interroge notre compréhension de la Révolution française et sur lequel nous insisterons ici, est précisément centré sur les limites, voire l'impossibilité, de toute restitution de la réalité historique ${ }^{2}$ :

Peut-être espère-t-il qu'en écrivant son aventure il s'en dégagera un sens cohérent.

Tout d'abord le fait qu'il va énumérer dans leur ordre chronologique des 
événements qui se bousculent pêle-mêle dans sa mémoire ou se présentent selon des priorités d'ordre affectif devrait, dans une certaine mesure, les expliquer. Il pense aussi peut-être qu'à l'intérieur de cet ordre premier les obligations de la construction syntaxique feront ressortir des rapports de cause à effet. Il y aura cependant des trous dans son récit, des points obscurs, des incohérences même. (Les Géorgiques, p. 8483)

2 À nos esprits qui semblent aujourd'hui de nouveau désireux d'épopée nouvelle - dans un contexte narratif ainsi défini : « ce que dit et fait la Révolution dans et à l'imaginaire d'un présent continu qui invite à relire le passé à sa lumière et à voir le futur à travers lui ${ }^{4} »-$, les textes de Simon, plutôt qu'ils ne proposent, soulèvent un problème, ce qui déjà crée une distance.

3 En quoi entrent-ils dans le sujet? Pour rappel, la famille maternelle de l'auteur, avec notamment ses liens la rattachant à un passé révolutionnaire, est au centre de La Route des Flandres (1960), des Géorgiques (1981), de L'Acacia (1989). Elle inspire aussi L'Herbe (1958), puis Histoire (1967) et encore l'ultime roman: Le Tramway (2001). Les Géorgiques roman d'archives, roman fait à partir d'archives, sont une clé pour le sujet qui nous préoccupe, Claude Simon étant notoirement - c'est l'objet de ce récit - descendant d'un Conventionnel ayant voté la mort du roi, issu de la petite noblesse des campagnes et devenu général d'Empire: Jean-Pierre Lacombe Saint-Michel (1753-1812). C'est avec une masse de vieux papiers hérités de cet ancêtre que Claude Simon s'est mis à écrire son roman. Dans celui-ci, les documents d'archives sont quasiment élevés au statut de personnages, ou plus exactement de motifs à peindre, à combiner, sur la surface de la page, on aurait envie de dire de la toile - Simon a été peintre, ayant étudié le dessin à l'académie d'André Lhote dans les années 1930 et pratiquant aussi la photographie durant toute sa vie ${ }^{5}$. Il a souvent expliqué en quoi, chez lui, le romanesque de la forme, de la langue, de la description prime avant tout, le jeu des associations et correspondances prévalant sur l'action proprement dite et les relations entre les personnages, toute narration psychologique étant aussi exclue ${ }^{6}$. C'est pourquoi les romans de Simon ne rendent pas compte d'un temps fait de moments qui se succèdent, mais du temps fait de moments simultanés. L'écoulement du temps est représenté de manière visuelle, comme une étendue, ce qui a pour effet d'en donner une image figée :

Des lois peut-être (un ordre ou plutôt une ordonnance impossible à détecter mais d'une nature aussi imprescriptible, aussi mathématique, que celles qui président aux spirales des coquillages, organisent en étoiles les cristaux de neige ou structurent les plus infimes particules vivantes) voulaient-elles que les diverses phases, les divers stades du processus (ou du cérémonial) qui s'était déclenché fussent observées. (Les Géorgiques, p. 729)

Il ne faudrait pas pour autant que les historiens s'en détournent, en dépit du relatif inconfort qu'ils peuvent ressentir à l'égard de ces textes, se contentant de se mêler à la voix publique, quelque peu paresseuse parfois, pour laquelle les romans de Simon seraient réputés un peu difficiles à lire 7 .

Pour parler de cette relation que Simon entretient avec la Révolution française à travers ses romans, par le moyen de ses romans, il faut suivre trois chemins qui indiquent autant de répliques portées par l'écrivain face à trois injonctions avec lesquelles il se débat de livre en livre. Il est important de considérer qu'il y a là ressassement, signe que cette obsession déborde sans cesse la production de chacun des textes, et est à chaque fois remise en jeu. Une écriture de derrière une épaisse paroi de verre d'abord, face à l'injonction de la représentation; l'événement n'étant disponible 
qu'à l'état d'archive - sorte de pare-feu à l'injonction généalogique ; la recherche d'un moi historiquement situé enfin, face à l'injonction de la mémoire.

\section{L'écriture de derrière l'épaisse paroi de verre}

Dans un avant-texte, un brouillon de travail des Géorgiques, inédit, on peut lire :

Il perçoit tout comme à travers une vitre (développer : aquarium) -éléments aux particularités différentes, densité du son, liquide etc... Impression d'être déjà ailleurs. Le Temps que mettent à lui parvenir les aboiements du chien (comme s'ils avaient à traverser une épaisse paroi de verre - ou de temps : lui parvenant déjà d'un autre monde renforce ce sentiment ${ }^{8}$.

7 Un texte de Claude Simon se reconnaît par sa manière de rouvrir le dispositif même de la représentation - et par-là même le concept - exactement comme on ouvre un corps pour une opération chirurgicale, en considérant que le préfixe « re- » qui entrouvre le mot de l'intérieur, indique une syncope, rappel de la part d'intransitivité dans le langage. C'est d'emblée mettre en perspective la part de l'absence et de la présence. C'est poser aussi la dimension réflexive de l'imagination autour des modalités de ses mécanismes mêmes. C'est engager une critique des schèmes culturels incorporés en tant que formes institutionnalisées des modes de régulation, de contrôle, de domination de la faculté de penser.

Simon est né en 1913, son écriture s'inscrit dans l'ère des lendemains de la catastrophe des années 1939-1945. À sa manière, il a participé à une révolution littéraire, mais sans jamais abdiquer sa singularité, voire son hétérodoxie, vis-à-vis du Nouveau Roman et son rejet de la fonction référentielle de l'écriture ${ }^{9}$. Il était étranger à toute version doctrinale des esthétiques dans lesquelles il s'engageait. Il désirait conserver malgré tout sa liberté vis-à-vis des conceptions de l'écriture qui donnent l'exclusive à la seule dynamique du langage en tant que tel. Mais Claude Simon a dit, il est vrai, son hostilité au témoignage, à ses yeux toujours sujet à caution. Il n'a eu de cesse de le tourner en dérision; c'est pourquoi il s'est écarté de toute écriture à la première personne du singulier, qui semblerait ramener le roman à une fonction documentaire ${ }^{10}$. Il a adopté une recherche ascétique et laborieuse de ce qui fait la construction d'un texte, y intégrant les obstacles rencontrés au sujet même de sa réflexion. Il se sentait aussi irrémédiablement hanté par la scène capitale où il avait failli, dragon dérisoire sur son cheval, perdre la vie à la frontière belge, en pleine déroute militaire en mai 1940 face aux panzers de Rommel - motif de nouveau repris dans la deuxième partie des Géorgiques, les époques se répondant dans un jeu d'échos. D’où la figure récurrente autant qu'obsédante du cavalier qui bascule à terre chez Simon et qui s'affronte à un réel pour lequel les mots ont perdu leur pouvoir de signifier. Quelque chose s'est joué, ce jour-là où il faillit perdre la vie, de son rapport aux images : désillusionné pour toujours, ses yeux meurtris et son regard désabusé seraient dorénavant attirés par ce qui troue et tache les mécanismes de la représentation. Après bien d'autres, Simon retissa ensuite les liens de connivence entre la littérature et la figure de la chute, en faisant de cette dernière son monde, son expérience de l'appréhension des contingences, du tangible, des limites ${ }^{11}$. La chute aura ouvert une entreprise de connaissance concernant son sentiment de la finitude à l'occasion de ces situations où est rappelée une non-coïncidence à soi, un déterminisme irréductible occulté :

Il éprouve la sensation d'être séparé du monde extérieur par la pellicule craquelée

et brûlante que forment sur son visage non seulement la saleté mais encore son état 
d'extrême épuisement. Il lutte tant bien que mal contre le soleil qui alourdit ses paupières, oscillant d'avant en arrière sur sa selle au pas de son cheval. (Les Géorgiques, p. 676).

Les textes de Simon ne font pas moins circuler des discours politiques comme rappels du contexte englobant, mais ceux-là agissent comme des motifs discursifs placés à l'arrière-plan. Ils sont de surcroît dominés par l'éternelle lutte transhistorique des passions et des sentiments ${ }^{12}$ :

Plus tard, il se lança dans des explications compliquées et pour ainsi dire techniques, émaillées de sigles, d'initiales de partis, de syndicats, de factions, d'organismes de police, de ligues ou d'unions, comme les symboles de corps chimiques seulement compréhensibles aux initiés et qui, selon la façon dont ils sont mélangés et dosés, peuvent se combiner à peu près à l'infini pour constituer aussi bien des engrais, des détergents ou des explosifs. (Les Géorgiques, p. 877)

En passer par un dé-savoir, une dé-formulation, et décrire les filtres de la mémoire et du langage ${ }^{13}$; la réalité est à jamais perdue, la lacune impose un deuil de la causalité ; pas de mise en intrigue, pas de restitution globale ; une reconsidération de la logique $\mathrm{du}$ monde et du processus d'élaboration du sens ${ }^{14}$. Ce qui se bouscule dans la conscience, fragments d'un grand magma désordonné, entrave toute prétention revendiquée par la narration omnisciente, soi-disant capable de restituer un quelconque enchaînement causal et continu. Déformation mémorielle préalable donc, redoublée par celle qu'apporte en elle-même l'écriture. Les éléments biographiques ne sont alors qu'un prétexte, à ne pas confondre avec le texte qui les mobilise :

Rien ne sort de rien, et il est moins paradoxal qu'il n'y paraît à soutenir que tout écrit est autobiographique. Qu'il décrive un événement ou un personnage, un objet « réel » ou fictif, un écrivain ne fait toujours que se décrire lui-même, c'est à dire au présent de l'écriture ${ }^{15}$.

11 Toute vie écrite introduit donc un écart. Les documents réunis par le romancier pour écrire véhiculent eux-mêmes un doute sur la véracité des faits rapportés. L'ensemble des Géorgiques repose précisément sur ce principe. Ce voile et ce soupçon que produit l'écriture correspondent à la démarche de Claude Simon, c'est à ce titre que les documents, délestés de leur fonction de preuve, ont pu venir sans peine s'inscrire dans le fil de sa narration.

12 Le roman a donc méticuleusement construit le lieu du manque; en cet endroit entrent les documents d'archives, comme un privilège donné à une matière faite de rebuts déplacés par une houle qui elle-même s'est retirée. Les documents attestent seulement d'une béance irréversible qui met en déroute toute lecture critique; il devient impossible de les interpréter, ils sont anachroniques, devenus incapables de se référer à une réalité démontrée qui n'importe plus pour elle-même ${ }^{16}$. C'est par contre leur combinatoire qui signifie, faisant passer de l'un à l'autre, l'action de l'histoire qui s'écrit n'en étant que le produit. Tel est le rythme donné à l'ensemble du roman, singulièrement au moyen d'une succession haletante et vertigineuse qu'entonnent par exemple les premières lignes de la première partie des Géorgiques, et pour mettre le lecteur dans les conditions qui lui permettront de se figurer comment sera décrit dans ce texte le révolutionnaire désigné sous le sigle L.S.M. :

Il a cinquante ans. Il est général en chef de l'artillerie de l'armée d'Italie. Il réside à Milan. Il porte une tunique au col et au plastron brodés de dorures. Il a soixante ans. Il surveille les travaux d'achèvement de la terrasse de son château. Il est frileusement enveloppé d'une vieille houppelande militaire. Il voit des points noirs. Le soir il sera mort. Il a trente ans. Il est capitaine. Il va à l'opéra. Il porte un 
tricorne, une tunique bleue pincée à la taille et une épée de salon. (Les Géorgiques, p. 655).

13 Par-delà le ton péremptoire des phrases à la forme affirmative, c'est dire que la fiction ici n'est pas un recours, mais le propre de la méthode, résultat du travail de l'écriture dans le langage, ou pour mieux dire de la mobilité des mots, leurs polysémies, les nœuds de significations qu'ils signalent. Pour Simon, il n'y a pas de défaut de connaissance auquel il faudrait remédier ; il y a qu'un défaut de reconnaissance de la consistance linguistique de l'imagination.

Parce qu'il m'a fallu du temps pour écrire Les Géorgiques. J'étais en possession d'une montagne de papiers laissés derrière lui par l'un de mes ancêtres, le personnage que j'appelle L.S.M., et j'avais envie de faire quelque chose de ces archives, mais je ne savais comment m'y prendre : je veux dire passer de ces documents bruts à la littérature, composer une œuvre littéraire qui les englobe et les dépasse. Cela posait de gros problèmes. [...] De même que tous ces vieux papiers de famille n'ont été pour moi qu'un ferment [...]. Comme je vous l'ai dit, c'est un « bricolage » à partir de papiers de cet ancêtre dont l'histoire m'intriguait. Puis il s'est développé peu à peu, sans que je sache exactement où j'allais. C'est toujours ma manière de faire. [...] Vous savez, je crois qu'écrire, c'est avant tout composer: agencer les mots à l'intérieur de la phrase, les phrases à l'intérieur des paragraphes, les motifs à l'intérieur du texte... Les Géorgiques une fois écrites, je les ai entièrement recomposées ${ }^{17}$.

14 Quand Simon dit fiction, il pense langage ${ }^{18}$, toute réflexion sur la compréhension du monde engageant une science du langage. Il est ici un héritier de Mallarmé ${ }^{19}$, pour qui «toute méthode est une fiction », « le langage se réfléchissant », la science aux yeux du poète n'ayant pas d'extériorité par rapport au langage, celui-ci n'étant nullement un moyen mais une fin $^{20}$. Simon se fait aussi sur ce point, afin de souligner la part d'intransitivité du style, lecteur notoire du Degré zéro de l'écriture de Barthes, souvent cité dans des entretiens tout comme les formalistes russes qui sont redécouverts en France dans les années $1960^{21}$ :

Et si, suivant Chlovski, on s'accorde à définir le «fait littéraire » comme «le transfert d'un objet de sa perception habituelle dans la sphère d'une nouvelle perception ", comment l'écrivain chercherait-il à déceler les mécanismes qui font s'associer en lui ce «nombre incalculable» de «tableaux» apparemment « détachés» qui le constitue en tant qu'être sensible, sinon dans cette langue qui le constitue en tant qu'être pensant et parlant et au sein de laquelle, dans sa sagesse et sa logique, nous sont déjà proposés d'innombrables transferts ou transports de sens $^{22}$ ?

\section{L'événement n'est disponible qu'à l'état d'archive}

Pourtant le roman s'est laissé envahir par les archives ; celles-ci ont charrié certes de l'inconnaissable, mais qui n'attendrait qu'à être déchiffré. L'auteur n'est pas non plus le maître du jeu qu'il a voulu à son tour établir. D'un côté, Les Géorgiques prennent acte d'un traumatisme inhérent à tout discours sur l'Histoire avec une majuscule, et abandonnent toute synthèse qui entendrait réconcilier les temporalités.

[...] l'Histoire se mettant à tournoyer sur place, sans avancer, avec brusque retours en arrière, d'imprévisibles crochets, errant sans but, entraînant tout ce qui se trouvait à la portée de cette espèce de tourbillon, le happant lui-même au passage, par surprise, le projetant (ou le précipitant) au centre du même maelström [...]. (Les Géorgiques, p. 899) 

entendre son altérité qui le fait différer d'un présent de la narration tout occupé, lui, à rompre avec l'idée de transparence de la rétrospection ${ }^{23}$. Le roman généalogique que poursuit Claude Simon tout au long de son œuvre fait alors éclater les cadres du genre autobiographique. Il amplifie les manques et les secrets. Il témoigne d'une insatisfaction permanente qui motive une incessante récriture. D'un roman à l'autre, les variantes de l'histoire familiale réinventent et reconstruisent les dates et les lieux. Elles ajoutent autant qu'elles suppriment. Elles transforment, travestissent, questionnent les racines et les legs des générations antérieures. L'œuvre de Claude Simon est hantée par la généalogie, mais sur le mode d'une remise en cause et du soupçon. Le non-dit, le non-explicite, le sous-entendu et le silence désignent une architecture sous-jacente de l'écriture. C'est pourquoi le lecteur se trouve particulièrement sollicité pour interpréter à son tour une combinatoire complexe de motifs qui se répondent d'un texte à l'autre.

La parution des Géorgiques fût décisive pour la critique ${ }^{24}$. L'insertion ostensible d'archives dans le texte réaffirmait sans détour la fonction référentielle d'une écriture perméable à un en-dehors que, finalement, elle convoquait bel et bien. Elle commandait d'interroger de nouveau le fonctionnement interne du texte, capable autant de produire sa propre réalité que d'accueillir et de réinterpréter l'articulation entre histoire familiale et histoire collective ${ }^{25}$. Si l'on pouvait toujours y lire une poétique singulière de l'archive, qui travaille autant avec une documentation qu'avec les lacunes de celle-ci, au service de formes nouvelles, le texte exhibait un rapport ambivalent et instable avec un réel à la fois désigné et fuyant ${ }^{26}$. Georges Raillard fut le premier à pouvoir, dès 1987, comparer le texte des Géorgiques et les archives Lacombe SaintMichel grâce à la possibilité que lui offrit Claude Simon d'accéder aux papiers de son ancêtre. Il concluait que «le style du Général est une création simonienne ${ }^{27}$ ». Effacement, changement, coupure, fragmentation, collage, dans Les Géorgiques, la rhétorique de l'ancêtre, son esthétique, son emphase avaient été en partie recomposées mais pas totalement, plus exactement raccourcies, ou résumées, ou encore transformées par le truchement du discours indirect libre. Paradoxale exhibition des papiers de l'ancêtre, en réalité tronqués. Le roman ne reproduisait pas le document tel quel, il se l'appropriait, le digérait, le régurgitait.

Maurice Halbwachs l'avait souligné, en son temps, avec force : «Au reste, comme les croyances générales d'une société parviennent aux membres des familles par l'intermédiaire de ceux d'entre eux qui sont le plus directement mêlés à la vie collective $\mathrm{du}$ dehors, il peut arriver ou bien qu'elles soient adaptées aux traditions de la famille, ou inversement, qu'elles transforment ces traditions ${ }^{28} »$. Les Géorgiques font en effet de nouveau surgir le motif de la culpabilité ancestrale qui se retrouve dans nombre des romans de Claude Simon ${ }^{29}$. Malheur qui se transmet de génération en génération et qui renvoie chaque personnage à son propre destin. La famille semble souffrir d'un mal héréditaire et l'héritage porte la marque d'une malédiction. La famille est menacée par une faute inexpiable qui remonte à ses origines. Une caste, par son hybris, s'attire les foudres de la Providence. Pour se préserver, elle développe sa propre mythologie intime, avec ses secrets inaccessibles.

[...] comme si la vieille dame s'infligeait à elle-même on ne savait quelle pénitence, assise là, devant ces assiettes au contenu desquelles elle ne faisait que goûter, perdue dans la contemplation d'une suite de désastres dont l'épidémie de phylloxéra devait obscurément lui paraître, avec la série de deuils qui avait frappé 
la famille, comme le couronnement, quelque vengeance du sort, l'inéluctable lointain châtiment d'une fortune édifiée dans le tumulte et une violence qu'elle se considérait peut-être condamnée à expier sans fin et même assumer [...]. (Les Géorgiques, p. 762)

19 Une vérité ne doit pas être divulguée. Le narrateur poursuit une enquête, au-delà du visible; il combat avec sa propre conscience un discours officiel qui dissimule déterminations et intentions. Le roman se confronte à la mémoire sociale collective, il entend déjouer la tromperie d'un monde qui a dénaturé les idées, il dénonce les leurres légués par une écriture coercitive qui censure la compréhension du passé. Il désirerait changer le rapport que l'homme entretient avec le langage ${ }^{30}$. Il intente un procès en défiance vis-à-vis du monde des apparences, et ouvre une crise de l'authenticité et de la restitution médiatisée par des supports culturels, simulacres de vérités ${ }^{31}$. Il espère une place pour le récit qui n'aurait pas été assignée.

On dirait que les mots assemblés, les phrases, les traces laissés sur le papier par les mouvements de troupes, les combats, les intrigues, les discours, s'écaillent, s'effritent et tombent en poussière, ne laissant plus sur les mains que cette poudre impalpable, couleur de sang séché. (Les Géorgiques, p. 692)

20 Le texte de Simon ne dissimule nullement le filtre qu'opère le personnage fictif du descendant lointain du Général, quand il déchiffre ces étranges manuscrits venus du passé. C'est cette réception qui est en elle-même questionnée, telle une perception oblique, qui produit des altérations de la compréhension, une transmission en réalité non linéaire, l'inverse d'une reproduction. Ce sont des ruines d'archives qui sont décryptées par la narration. Le roman donne à lire des vestiges lacunaires :

Lorsque l'on tourne les pages des registres en les tenant inclinés de fines particules couleur rouille aux facettes scintillantes et dorées comme du mica se détachent des lettres et glissent sur les feuilles. (Les Géorgiques, p. 692)

21 Toute restitution du passé est impossible compte tenu de l'imagination, du travail de réinvention par la mémoire, des contraintes du langage; autant d'obstacles à toute volonté d'imitation mais autant de ferments de l'écriture.

Comme Proust l'a dit, "la seule réalité est la réalité écrite », que ce soit dans le moule des mots, des couleurs, des formes ou celui des symboles mathématiques et, hormis le toucher, nous ne connaissons des choses que ce qui nous en est dit. C'est en n'étant "tout occupées que d'elles-mêmes", selon les paroles de Novalis, et à cette condition expresse, que l'art, la littérature et la science participent à l'incessante transformation du monde, à cet incessant mouvement, et si leurs « crises » prenaient fin, cela signifierait que le monde s'est arrêté de tourner ${ }^{32}$.

L'invention d'une famille fictive romanesque introduit un jeu de proximité et de distance. Le personnage récurrent d'oncle Charles, par exemple, superpose en partie la figure d'Henri Carcassonne, marié à la sœur de la mère de Claude Simon, et celle de Paul Codet, tuteur du futur écrivain à l'époque de sa scolarité parisienne ${ }^{33}$. Charles supervise les versions latines de son neveu dans Histoire, et prolonge son rôle d'éducateur dans Les Géorgiques en mettant sous les yeux du narrateur les documents d'archives de l'ancêtre Conventionnel :

[...] la voix de l'oncle Charles disant: «Ils sont à ta disposition si ça t'intéresse. Peut-être es-tu encore trop jeune, mais plus tard... Quand tu seras vieux toi-même. Je veux dire quand tu seras capable non pas de comprendre mais de sentir certaines choses parce que tu les auras toi-même éprouvées... C'est à la fois fastidieux et fascinant. » (Les Géorgiques, p. 938) 
famille se situe entre le Moi et le monde, elle offre un modèle réduit de l'Histoire universelle et renvoie aux tumultes d'événements qui ne sont qu'apparemment révolus. Dans Les Géorgiques, la maison, symbole littéraire magnifié de la lignée, renferme des reliques tantôt dissimulées dans ses placards (les archives du quadrisaïeul L.S.M.) et ses tiroirs (un document éclairant le conflit entre le Général républicain et son frère ayant rejoint la contre-révolution), tantôt exhibées dans ses salons qui ne sont ouverts qu'à l'occasion (le buste de l'ancien Conventionnel). L'enquête porte sur le mythe de l'ancêtre ; la quête du passé examine les traces qu'il aurait laissées. Déjà dans Histoire et dans L'Acacia, l'arbre offre une métaphore des ramifications labyrinthiques qui démultiplient les relations de filiations, fouillis occultant ce que l'on ignore, délaisse ou oublie.

Dès 1961, Simon déclarait dans un entretien: «Mais j'ai retenu assez d'histoires de famille, de ma famille, pour emplir longtemps mes livres ${ }^{34}$.» C'était dire d'emblée que l'écriture sert aussi à s'offrir des ancêtres choisis et triés sur le volet, à soulager la pesanteur d'une conception cyclique de l'Histoire, où l'héritier semble condamné à la répétition de la geste paradigmatique d'une tradition familiale. Les mots en eux-mêmes servent à découvrir et interroger des parentés plus ou moins indirectes. Un roman comme Les Géorgiques est entièrement structuré autour de la relation analogique entre les temps, les lieux, les hommes, les choses : entrent en résonance la Révolution, la guerre d'Espagne, la débâcle de mai-juin 1940. L'écriture construit des intersections puis réunit des éléments plus ou moins communs dans un ensemble plus large, elle trouve sa motivation à travers de multiples passages et conduits qui finissent par former une nébuleuse dynamique. La phrase explore des carrefours qui en même temps la traversent de part en part. Campagne, champ, terrain, division réfèrent autant à la guerre qu'à l'agriculture ${ }^{35}$ :

Je veux parler de cet éternel recommencement, cette inlassable patience ou sans doute passion qui rend capable de revenir périodiquement aux mêmes endroits pour accomplir les mêmes travaux : les mêmes prés, les mêmes champs, les mêmes vignes, mes mêmes haies à regarnir, les mêmes clôtures à vérifier, les mêmes villes à assiéger, les mêmes tranchées périodiquement ouvertes sous les mêmes remparts: Coblence, Pavie, Namur, la Meuse, Mantoue, l'Yssel, Anvers, l'Adige, Vérone, Peschiera, Mayence... Prises, franchies, perdues, reprises, reperdues, reconquises de nouveau reperdues encore, infatigablement, sans fin ni espoir de fin, avec pour seules variantes les prévisibles imprévus des successives coalitions de la pluie, du gel ou de la sécheresse... (Les Géorgiques, p. 939)

Les documents d'archives qui ont servi à l'écriture des Géorgiques éclairent l'idéologie lignagère de la famille maternelle de Claude Simon, mais aussi le désir de l'écrivain de s'en départir et d'afficher une farouche et ironique volonté de désaffiliation. Ils composent à leur manière une étroite imbrication entre mémoire familiale et mémoire sociale collective à partir de laquelle le romancier a lui-même mené sa propre introspection.

À l'arrière-plan de l'œuvre se dessine en effet une même caste, avec ses marques de mondanités ${ }^{36}$. Le narrateur nourrit une certaine sympathie à l'égard de ses ancêtres, surtout ceux avec lesquels il peut s'identifier en traversant des expériences qui lui apparaissent similaires, si ce n'est communes. De génération en génération, les hommes auront été formés à la violence du métier des armes, ils auront connu l'épreuve des batailles, ils auront connu une vie aventureuse, et les jeunes épouses n'auront pas eu d'autres choix que d'attendre leur retour. Tout l'imaginaire parental 
que véhiculent les romans de Claude Simon instruit une relation dédoublée vis-à-vis de la figure paternelle, figure autant réelle que symbolique, faite d'attachements et de déprises, et déclinée selon ses multiples variantes: Reixach de La Route des Flandres, Henri d'Histoire, L.S.M. des Géorgiques, l'officier de L'Acacia, oncle Charles enfin de roman en roman.

\section{Le moi historiquement situé}

27 La critique a relié l'écriture des Géorgiques au ressaisissement rétrospectif par Claude Simon de la dimension autobiographique de l'ensemble de son œuvre ${ }^{37}$. N'avait-on pas défendu antérieurement, à grands renforts des déclarations de l'écrivain, que l'écart entre sa vie et son écriture allait grandissant? N'avait-on pas cru à l'exploration de la dynamique irréductible de la langue?

La préparation des Géorgiques s'est accompagnée effectivement d'une mise au point qui soulignait une ambivalence en réalité toujours présente depuis le début, de roman en roman, que l'écriture se chargeait plus explicitement encore de travailler : «En fait, ce que je fais de la langue en la travaillant, c'est-à-dire mon langage, me constitue en tant que sujet historique ${ }^{38}$."

Ce moi historiquement situé et produit de l'écriture, c'est celui qui restait tout autant relié à une documentation, à un matériau dont l'exploitation allant à son tour grandissante, en grande partie héritage familial recueilli et stimulateur d'un rapprochement entre la famille fictive des romans, faite de personnages récurrents, et la propre famille de l'écrivain. Du coup, c'est la hantise du lignage qui apparaissait après coup comme un motif fédérateur de la volonté d'écrire chez Claude Simon, vrai élément de continuité à partir duquel le « vécu » s'écrit.

Il semblerait bien que le jeune Claude Simon se soit montré déjà grandement passionné par les récits de la Révolution française et du ${ }^{\mathrm{er}}$ Empire, par le rôle joué par son ancêtre ${ }^{39}$. La révolution comme processus aura été pour lui un domaine sérieux, intéressant. Jeune adulte, il s'était montré concerné par les bouleversements politiques qui affectaient le monde, jusqu'à une très brève adhésion au PCF en 1937, essentiellement pour pouvoir voyager dans l'Espagne de la guerre civile et l'URSS de 1937. Il notait dans un carnet inédit au sortir de son séjour en Russie soviétique en juin 1937 : « Révolution par désespoir et négation ${ }^{40}$ ». Grand lecteur de Dostoïevski, son idéal révolutionnaire touchait davantage à la morale qu'à la politique. Reste qu'il est toujours possible de puiser chez lui quelques formulations explicites, et fort communes, tendant à identifier l'idée de littérature à celle de révolution ${ }^{41}$. En 1967, il a 54 ans : « Mais c'est seulement s'il obéit exclusivement à son dieu, en d'autres termes au langage, que mon dire, alors, concernera tous les hommes et pourra peut-être ainsi s'inscrire dans la révolution toujours recommencée du monde. ${ }^{42}$ "

31 Simon a été marqué par le retournement idéologique des années 1920-1930 durant lesquelles la croyance en l'effondrement du capitalisme et l'avènement d'une révolution en Occident a été démentie par la montée du fascisme, la division du communisme, l'aporie à faire se rejoindre la révolte de l'esprit et la révolution prolétarienne ${ }^{43}$. Claude Simon a connu l'effondrement du grand récit révolutionnaire et réfléchi à sa manière à la déception engendrée par celui-ci. L'expérience traumatisante des guerres, finalement plus que celle des révolutions, aura pour lui contribué à forger 
son jugement éthique comme son esthétique. Sa sympathie allait aux victimes et aux vaincus.

Si, comme nombre de ses contemporains proches ou semblables, il a été amené à cesser d'accorder à la révolution politique le rôle de moteur dans sa démarche intellectuelle ${ }^{44}$, il n'en a pas moins toujours témoigné d'une méfiance bien ancrée, assez typique de l'héritage des avant-gardes de l'entre-deux-guerres, à l'endroit de l'humanisme bourgeois, de toute forme d'union sacrée, et des mouvements exclusivement littéraires. Claude Simon a dit son hostilité à toute transcendance, le monde n'est que ce qu'il est ${ }^{45}$. Il a rapporté d'un court séjour dans la Barcelone de 1936, en armes et déchirée entre factions, un irrémédiable pessimisme, bien qu'il restât très impressionné par ce qu'il y a $v^{46}$. Il fut parmi les signataires du Manifeste des 121 en juillet 1960 sur Le droit à l'insoumission dans la guerre d'Algérie, et à ce titre inculpé. Il fut comme d'autres cité à comparaître quelques mois plus tard comme témoin en faveur de certains des accusés lors $\mathrm{du}$ procès du "réseau Jeanson", soutien du FLN, attentif à la révolutiondécolonisation qui cette fois-ci se déroulait hors de l'Europe, et même en partie contre elle et ses propres schémas politiques. Il a pu ainsi éprouver comment, en 1960, une idée révolutionnaire pouvait se retrouver sur le banc des accusés, frappée d'illégitimité politique par les gouvernants qui dirigeaient les destinées de son propre pays. Son texte servant d'auto-défense, longtemps inédit, a été publié récemment ${ }^{47}$; il arguait de sa position d'intellectuel, et en appelait aux principes fondateurs du libéralisme, à ses yeux incompatibles avec le délit d'opinion. Rarement, pour Simon, l'écrivain a été aussi proche du citoyen.

L'attribution du prix Nobel en 1985 à Claude Simon est venue confirmer le rapport ambivalent que l'écrivain entretenait avec l'humanisme. Si l'œuvre pouvait sembler dire l'impasse, si ce n'est la faillite, du projet émancipateur, progressiste et anthropocentré des Lumières, le lauréat n'en défendait pas moins la liberté de penser et la résistance à l'oppression, rappelant un de ses leitmotivs sur le pouvoir du langage de l'écrivain à changer, un tant soit peu, le monde ${ }^{48}$. Et, avec toute l'ironie qui le caractérise, Simon s'adressait au jury réuni à Stokholm en décembre 1985 pour le souligner :

Laissons de côté l'insinuation selon laquelle certains agents d'une police politique pourraient siéger parmi vous et vous dicter votre choix, quoique, au passage, il ne soit pas sans intérêt de remarquer qu'aujourd'hui encore, dans certains milieux, l'Union soviétique reste le symbole de redoutables forces de subversion auxquelles il est ma foi flatteur pour un simple écrivain de se voir associé, car enfin on a tellement, ici et là, dénoncé l'égoïste et vaine gratuité de ce qu'on appelle «l'art pour l'art » que ce n'est pas pour moi une mince récompense de voir mes écrits, qui n'avaient d'autre ambition que de se hisser à ce niveau, rangés parmi les instruments d'une action révolutionnaire et déstabilisatrice ${ }^{49}$.

Si l'on remonte quelque vingt ans plutôt, le texte paru dans L'Express en juillet 1963 sous le titre «Et si les écrivains révolutionnaires jouaient le rôle de la presse du cœur? $\aleph^{50}$, critique la littérature dite engagée. Mais il trouve son complément, presque son symétrique inverse, dans celui publié par La Quinzaine littéraire en mai 1967, » Tradition et révolution $»^{51}$, sur la fonction émancipatrice de la langue travaillée par l'écrivain au sein de toutes les tentatives d'ordre intellectuel visant à remettre en cause les conventions, citations de Marx à l'appui. Même critique, en janvier 1988 devant un parterre de Nobel, de toute intrusion d'un projet idéologique 
dans les arts, mais même confiance dans l'avenir d'un enrichissement intellectuel général ${ }^{52}$.

Sans doute, plus qu'auparavant encore, le lien intime entre le thème de l'impuissance de la culture à empêcher la dégradation de l'homme et celui de la difficulté de la transmission entre générations est apparu aux yeux de la critique comme le cœur d'une écriture plus engagée qu'il n'avait pu sembler ${ }^{53}$. C'est cette manière d'articuler la question de la valeur de la mémoire culturelle à celle de la légitimité de son héritage qui aura alors retenu l'attention :

Et si les bienfaits des arts et des lettres apparaissent de façon moins immédiate (il n'y a pas, en effet, de " progrès » en art), l'homme s'est cependant modifié chaque fois qu'un peintre, qu'un sculpteur ou qu'un écrivain, eux-mêmes issus de la longue suite de leurs prédécesseurs, ont dit le monde d'une façon tant soit peu nouvelle: même l'illettré qui n'a pas lu et ne lira jamais Flaubert, Rimbaud ou Joyce ne vit pas aujourd'hui de la même façon que son semblable avant que ceux-ci apparaissent ${ }^{54}$.

Pas de table rase possible pour l'héritier, mais une nécessité qui s'impose à lui : revendiquer l'appartenance autant que la contester. D'où un constant va-et-vient entre identification et distanciation, hommage et parodie, de personnage en personnage, celui du père notamment - saint-cyrien de la III République dans L'Acacia dont l'excellence du parcours doit tout à l'abnégation de ses sœurs institutrices laïques - et surtout l'ancêtre Conventionnel - variante d'un motif récurrent -, qui se suicide dans La Route des Flandres et meurt épuisé dans les Géorgiques.

La parution de ce dernier roman a même semblé infléchir significativement l'écriture en faveur d'un mouvement retrouvé de l'Histoire, avec une majuscule, une croyance en sa force impérieuse avec sa mécanique certes indéchiffrable et complexe mais somme toute logique, alors que La Route des Flandres la rendait responsable des illusions et des malheurs dont été avaient victimes les générations précédentes. Avec Les Géorgiques, l'Histoire et la Révolution ne semblaient plus dérisoires et la langue de l'écrivain continuait tout autant et comme avant à en explorer les variations ${ }^{55}$. L'entretien paru dans Le Monde du 4 septembre 1981 accompagnant la parution du roman clarifiait les choses, s'il en était besoin. Paroles de descendant d'un Conventionnel régicide :

- C'est la première fois que la Révolution française intervient dans votre œuvre. Quels sont vos sentiments envers elle et envers toute révolution?

- Le mot révolution est un mot valise. Il a servi à recouvrir - ou à masquer - tant de choses différentes sinon contraires, de la révolution quarante-huitarde à la révolution(!) islamique, en passant par la Commune, la révolution russe, la «Révolution nationale » de Pétain, la révolution «culturelle» de Mao... qu'il est bien difficile de répondre à votre question.

Pour nous en tenir à la grande Révolution française, celle de 1789, il me paraît que, en dépit de ses excès, elle reste la seule véritable révolution de l'histoire, rupture en profondeur avec l'ancien état des choses, et qui a permis l'essor du monde moderne.

- Et le côté sanglant de la Révolution?

- Écoutez: je ne suis naturellement pas partisan de la peine de mort, mais il me semble qu'il faut remettre les choses dans leur contexte : couper la tête d'un roi, d'un monarque de droit divin, c'était l'acte transgressif absolu, décisif. Imaginez le courage intellectuel qu'il a fallu pour en prendre la décision.

- Alors les Géorgiques un roman sur la grandeur et l'horreur des révolutions?

- Je lui aurais donné un autre titre. Non, ce sont des variations, au sens où l'on entend le mot en musique, sur ce qui lie l'histoire (les guerres et les révolutions qui la font aussi) et la terre ${ }^{56}$. 

classe d'origine est un motif récurrent. À chaque génération semble se répéter une prédisposition à l'écart et à la sédition. Nombre des personnages se situent du côté où leur caste ne les attend pas $^{57}$. Dans Les Géorgiques, le député de la Convention rompt avec la noblesse, son épouse quitte ses riches parents installés en Suisse pour s'exiler au fin fond de la campagne du Sud-Ouest de la France ; le frère, quant à lui, bien qu'il ait rejoint la contre-révolution, incarnerait une « inclination congénitale à la rébellion, à la transgression », ce qui l'aurait « poussé à violer la loi » ${ }^{58}$. Le descendant, cavalier de mai-juin 1940, découvre, effaré, l'étrangeté des archives laissées par ses aïeux. Tel est le vrai lien qui s'établit en parallèle et clandestinement entre des figures et des attitudes réfractaires parfois éloignées dans le temps et dans l'espace. De roman en roman, le narrateur s'affranchit progressivement des versions familiales dont il est le dépositaire; chaque reprise biographique marque autant de déprises autobiographiques, l'effet de distanciation l'emporte ${ }^{59}$.

Mais la désaffiliation se double d'une ambivalence, et c'est sans doute sur ce point que le propre trajet de Claude Simon se trouve mis en jeu au cœur de ses productions fictionnelles. On peut en effet reconnaître à l'écrivain une acuité sociologique remarquable, conditionnée par l'hétérogénéité des mondes dont sont issus ses parents : la paysannerie du Jura côté père, l'aristocratie languedocienne côté mère. Ce double héritage aura permis à Claude Simon de se préserver de toute annexion, de se réclamer de deux ethos sociaux, de formuler des combinaisons inédites pour appartenir tantôt à l'un, tantôt à l'autre des deux mondes - tout en cherchant toujours à se démarquer -, d'articuler, par exemple, l'ascèse que réclame la trajectoire d'ascension sociale côté paternel et un sens élégant et retenu de la distinction côté maternel ${ }^{60}$. Vivre certes en rentier retiré, mais pas en aristocrate mondain ; et si le père de Claude Simon semble avoir voulu rejoindre les valeurs de la classe de son épouse, son fils choisit le chemin inverse tout en ne pouvant pleinement réintégrer un quelconque lieu originel.

Devenir écrivain aura donc consisté à assumer autant la culture artistique patrimoniale de la bonne bourgeoisie languedocienne que celle lettrée et laborieusement acquise par le père boursier soutenu par ses sœurs institutrices au fin fond des montagnes jurassiennes ${ }^{61}$. C'est un des thèmes communs à L'Herbe (1958) comme à Histoire (1967). Claude Simon a lui-même parlé du sentiment de "mésalliance » entre ses parents que cultivait et affectait sa famille maternelle ${ }^{62}$. Ce couple désassorti renvoyait aussi au contraste entre les deux paysages qui ont baigné son enfance "à peu près aussi dissemblables que peuvent l'être, par exemple, la Suède et la Grèce ${ }^{63}$ » : la sécheresse de la plaine aux abords de Perpignan tout le long de l'année et les futaies humides des alentours d'Arbois durant les vacances d'été. Dans l'univers fictionnel du roman Le Tricheur, paru en 1945, cet antagonisme s'incarne en une Catherine Laverne, la dernière descendante d'une vieille famille désargentée, mariée avec un peintre, fils d'instituteur, gagné aux idées socialistes. Mme Laverne, sa mère, est une vieille femme qui se déplace avec une canne, héritière du nom de ses ancêtres législateurs révolutionnaires, elle révèle à sa fille l'existence d'une cachette dans un placard, derrière une tapisserie punaisée, où était dissimulé de l'argent: «Si jamais il y avait une révolution ou je ne

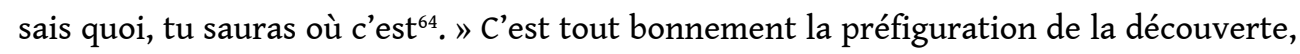
à la fin des années 1970, à l'occasion de travaux dans la maison familiale à Perpignan, de l'essentiel des papiers de l'ancêtre trouvés dans un placard d'escalier recouvert par 
des couches de papiers peints : nouveau matériau - un trésor - à déchiffrer pour se mettre à écrire Les Géorgiques.

Il y a là plus qu'une anecdote, car de livre en livre, les textes de Simon ne font pas que circuler des documents, parfois ici par prémonition, mais aussi des significations qui leurs sont préalables, plus ou moins avec la complicité de l'écrivain, mais plus ou moins parfois à son insu, chose par ailleurs sans doute assumée par lui. Il ne faut pas trop attendre d'un roman comme Les Géorgiques qu'il énonce des conclusions sur la Révolution française. Simon n'a pas d'ambition explicative à son propos. Tout au plus, plus globalement, il est de ceux pour qui révolution veut dire lutte contre l'idéologie, les conventions, idéologie révolutionnaire comprise s'il le faut. Ce qui est vrai, c'est que ses textes brisent l'épopée; la révolution - quel que soit le contexte historique où elle se déploie et revient - est à l'état de ruine, elle n'est plus un projet. On aura alors tôt fait d'identifier ses romans à une écriture du désenchantement typique de la deuxième moitié du Xx siècle.

Ce faisant, le motif de la révolution sous sa plume se voit tout autant rechargé d'une résonance languissante, à l'état en quelque sorte poétique, à l'exemple de l'état poétique du langage dont a parlé, il y a longtemps, Gérard Genette : soit un isolement, mais non pas à l'écart, bel et bien au milieu de l'arbitraire du langage courant, qui, en créant des marges de silence, donne à voir sur la page les mots et les images par un effet de retentissement et d'approfondissement ${ }^{65}$. La révolution est ici un signe, qui se manifeste par réminiscence, son décryptage n'est pas le but d'un roman comme les Géorgiques. C'est le fait qu'elle soit en cet état qui commande la réflexion sur le dispositif de sa représentation : la situation du lecteur est en quelque sorte analogue à celle des bergers d'Arcadie de la toile de Nicolas Poussin, quand est par exemple redécouvert pour lui l'inscription épitaphe sur le tombeau de l'épouse du personnage principal du roman. Ce monument funéraire, tout comme les documents écrits dont le roman tire toute sa sève, a une existence attestée, et sa description dans Les Géorgiques est le quasi décalque des conditions de sa révélation aux yeux de Claude Simon parti à sa recherche. C'est pourquoi un tel roman peut être mis en lumière - sans en épuiser l'ensemble des significations - par l'histoire familiale dont il découle. Il peut être de surcroît regardé en lui-même comme une archive supplémentaire venant s'ajouter à celles produites par la lignée, et dont il tire en partie sa substance. En ce sens ce n'est pas tant Claude Simon qui est l'héritier d'une révolution dont il pourrait disposer, mais bien plus elle - avec ses conséquences, son aura, sa mémoire - qui hérite par son intermédiaire, d'un écrivain. C'est seulement en ce sens que Simon entend exprimer son lyrisme à lui, et faire résonner ce qui réside en celui-là d'un écho révolutionnaire. Relisons une seconde fois son propos de 1967: «mon dire, alors, concernera tous les hommes et pourra peut-être ainsi s'inscrire dans la révolution toujours recommencée du monde ${ }^{66} . »$ 


\section{NOTES}

1.

Annales, histoire, sciences sociales, Savoirs de la littérature, 2010/2.

Critique, Historiens et romanciers. Vies réelles, vies rêvées ( $\left.n^{\circ} 767\right), 2011$.

Le Débat, L'histoire saisie par la fiction, (nº 165), mai-août 2011.

Littérature, Écrire l'Histoire, ( $\left.n^{\circ} 159\right), 2010 / 3$.

Roman 20-50, Actes. La portée de l'Histoire. Étude sur le roman français contemporain, 2011.

Romantisme, Histoire culturelle/Histoire littéraire, $\left(\mathrm{n}^{\circ}\right.$ 143) 2009/1.

Vingtième Siècle. Revue d'histoire, Histoire et roman, ( $\left.\mathrm{n}^{\circ} 112\right), 2011 / 4$.

2.

Celia BRITTON, « The Georgics : the limits of history », The Review of contemporary fiction, Spring 1985, vol. $5, \mathrm{n}^{\circ} 1$, p. 95-100.

3.

Claude SIMON, Euvres II, coll. La Pléiade, Paris, Gallimard, 2013. Sauf indication expresse, les références des pages des textes cités renvoient à cette édition.

4.

Extrait du programme diffusé pour notre colloque La Révolution en 3D-Textes, images, sons (1787-2440), 14, 15 et 16 mars 2019, université Paris 1 Panthéon-Sorbonne (IHMC-IHRF et CESPRA ; CNRS, EHESS, ENS, Paris 1).

5.

Voir Claude SIMON, Album d'un amateur, Rommerskirschen, Remagen-Rolandseck, 1988, avec 61 photographies de l'auteur, et Photographies 1937-1970, Maeght éditeur, Paris, 1992. De manière plus globale, sur ce sujet: Brigitte FERRATO-COMBE, Écrire en peintre: Claude Simon et la peinture, Grenoble, ELLUG, 1998.

6.

Claude SIMON, Quatre conférences, Paris, Minuit, 2012.

7.

Baudouin ESCHAPASSE, "Canular littéraire: Claude Simon est-il vraiment impubliable? Un manuscrit du Nobel de littérature, adressé à une vingtaine de maisons d'édition, a été refusé par la majorité d'entre elles. Faut-il s'en étonner?» Le Point.fr, 13 décembre 2017 [en ligne] https:// www.lepoint.fr/livres/canular-litteraire-claude-simon-est-il-vraiment-

impubliable-13-12-2017-2179532_37.php

8.

Bibliothèque Jacques Doucet, SMN Ms 12(2), feuillet 221.

9.

Voir le chapitre «... Et le Nouveau Roman. Singularité de Claude Simon », dans Mireille CALLEGRUBER, Claude Simon. Une vie à écrire, Paris, Seuil, 2011, p. 227-251.

10.

Jean H. DufFY, Notice du Jardin des Plantes, dans Claude SIMON, CEuvres I, coll. La Pléiade, Paris, Gallimard, 2006, p.1466-1489 ; Michèle TOURET, "Témoignage ", dans Dictionnaire Claude Simon, Textes réunis par Michel Bertrand, Paris, Honoré Champion, tome 2, 2013, p. 1046-1049.

11.

Laurent JENNY, L'expérience de la chute de Montaigne à Michaux, Paris, Presses universitaires de France, 1997.

12. 
Patrick REBOLARD, «Diégèses, palimpsestes, discours : dimensions et statuts du politique chez Claude Simon", dans Paul Dirkx et Pascal Mougin (dir.), Claude Simon: Situations, Lyon, ENS Éditions, p. 119-131.

13.

Mireille CAILle-Gruber, Le grand temps. Essai sur Claude Simon, Villeneuve d'Ascq, Presses universitaires du Septentrion, 2004.

14.

Alexandre DAUGE-ROTH, "Autobiographie et biographies parentales dans L'Acacia », dans Ralph Sarkonak (dir.), Claude Simon 2: L'Écriture du féminin/masculin, Revue des Lettres modernes, 1997, p. 127-152.

loc. cit.

15.

Claude SIMON, "Réponses de Claude Simon à quelques questions écrites de Roger-Michel Allemand », Une « Nouvelle Autobiographie », Le Nouveau Roman en questions, $\mathrm{n}^{\circ}$ 5, La Revue des Lettres Modernes, 2004, p. 238.

16.

Peter JANSENS, Claude Simon. Faire l'Histoire, Villeneuve d'Ascq, Presses universitaires du Septentrion, 1998.

17.

Entretien avec Jacqueline Platier, Le Monde des livres, 4 septembre 1981, p. 11, et p. 13.

18.

Claude SIMON, « La fiction mot à mot », dans Jean Ricardou et Françoise van Rossum-Guyon (dir.), Nouveau roman, hier, aujourd'hui, t. II, Paris, U.G.E., 1972, p. 73-97; ID., « Problèmes que posent le roman et l'écriture", Francofonia, $\mathrm{n}^{\circ} 18$, printemps 1990 ; et «Novelli ou le problème du langage », Les Temps Modernes, $\mathrm{n}^{\circ}$ 629, novembre 2004-février 2005.

19.

On notera le titre de la conférence en forme de citation prononcée en 1982 : «L'absente de tous bouquets ", dans Quatre conférences, Paris, Minuit, 2012, p. 39-71, ainsi que la référence explicite à l'occasion de la réception du prix Nobel: «[...] comme l'a dit Mallarmé, “chaque fois qu'il y a effort au style, il y a versification" ", Discours de Stockholm, 1986, dans CEuvres I, coll. La Pléiade, Paris, Gallimard, 2006, p. 897.

20.

Stéphane MALlarmé, "Notes sur le langage », dans CEuvres complètes I, coll. La Pléiade, Paris, Gallimard, 1998, p. 872.

21.

Entretien avec Madeleine Chapsal, L'Express, 10 novembre 1960 ; entretien pour La Revue de Paris, janvier 1961; entretien avec Madeleine Chapsal, L'Express, 5 avril 1962 ; «Il n'y a pas d'art réaliste ", entretien avec Madeleine Chapsal, La Quinzaine Littéraire, 41, 15-30 décembre, 1967. Entretien avec Jean-Paul Goux et Alain Poirson, « Un homme traversé par le travail », La Nouvelle Critique, $\mathrm{n}^{\circ}$ 105, juin-juillet 1977.

22.

Claude SIMON, Discours de Stockholm, op.cit., p.900. Écrire par «tableaux détachés » est une référence à Flaubert: voir Gisèle SÉGINGER, «Le Flaubert de Claude Simon», dans Écrivains contemporains de Flaubert, revue Oeuvres \& Critiques, XXXIV, 1, 2009, p. 65-86. La citation de Victor CHLOVSKI (1893-1984) est tirée de son texte "L'art comme procédé », traduit dans Tzvetan Todorov, Théorie de la littérature, Paris, Seuil, 1965, p. 76-97; dans ce texte, qui date de 1925, on relèvera notamment aussi cette affirmation : « Le but de l'image n'est pas de rendre plus proche de notre compréhension la signification qu'elle porte, mais de créer une perception particulière de l'objet, de créer sa vision et non pas sa reconnaissance. ", p. 89. 
23.

Jean-François HAMEL, «La poétique d'Orphée. Les révolutions de la mémoire historique chez Claude Simon ", dans Revenances de l'histoire. Répétition, narrativité, modernité, Paris, Minuit, 2006, p. $175-210$.

24.

Alastair B. DUNCAN (dir.), Claude Simon : New Directions, Edinburgh, Scottish Academic Press, 1985.

25.

Jean-Yves LAURICHESSE, «La critique simonienne et le référent. L'insistance du réel », dans Ralph Sarkonak (dir.), La réception critique. Claude Simon 6, Revue des lettres modernes, 2011, p. 97-133.

26.

Mireille calle-gruber, Mélina balCÁzAR moreno, Sarah-Anaïs CRevier, Anaïs fRANTz (dir.), Claude Simon. Les Vies de l'Archive, Dijon, Éditions universitaires de Dijon, 2014.

27.

Georges RAILLARD, «Les trois hautes fenêtres : le document dans Les Géorgiques de Claude Simon », dans Raymonde Debray-Genette et Jacques Neefs (dir.), Romans d'archives, Lille, Presses universitaires de Lille, 1987, p. 137-174.

28.

Maurice HALBWACHS, "La mémoire collective de la famille ", dans Les cadres sociaux de la mémoire (1925), rééd. 1994, Paris, Albin Michel, p. 168.

29.

Alexandre PRSTOJEVIC, Le roman face à l'histoire. Essai sur Claude Simon et Danilo Kis, Paris, L'Harmattan, 2005.

30.

Francine DUGAST-PORTES, «Le spectre de l'ascendance: fonction tragifiante du personnage de l'ancêtre au fil de l'œuvre de Claude Simon ", Revue des Sciences Humaines, ${ }^{\circ}{ }^{91}$, juillet-septembre, 1989 , p. 201-220

31.

Dominique VIART, Une mémoire inquiète. La Route des Flandres de Claude Simon, Villeneuve d'Ascq, Presses universitaires du Septentrion, 1997.

32.

Claude SIMON, « Problèmes que posent le roman et l'écriture », Francofonia, nº 18, printemps 1990, p. 10.

33.

Mireille CALLE-GRUBER : Claude Simon. Une vie à écrire, Paris, Seuil, 2011, p. 29-54.

34.

Entretien pour La Revue de Paris, janvier 1961, p. 139.

35.

Lucien DÄLLENBACH, «L'analogie généralisée », dans Claude Simon, Paris, Seuil, 1988, p. 127-138.

36.

Francine DUGAST-PORTES, « Le spectre de l'ascendance... », art cité.

37.

Alastair DUNCAN, "Claude Simon: le projet autobiographique", Revue des Sciences Humaines, $\mathrm{n}^{\circ}$ 220, 1990/4, p. 47-62 ; Une "Nouvelle Autobiographie», Le Nouveau Roman en questions, $\mathrm{n}^{\circ}$ 5, La Revue des Lettres Modernes, 2004.

38.

Entretien avec Jean-Paul Goux et Alain Poirson, « Un homme traversé par le travail », art. cité, p. 39.

39. 
D'après le témoignage recueilli par Mireille CALLE-GRUBER dans Claude Simon. Une vie à écrire, op. cit, p. 45.

40.

Ibid., p. 103.

41.

Laurent JENNY, Je suis la révolution. Histoire d'une métaphore (1830-1975), Paris, Belin, 2008.

42.

Claude SIMON, « Littérature : tradition et révolution », La Quinzaine littéraire, 27, 1-15 mai 1967.

43.

Nicole RACINE, "Une revue d'intellectuels communistes dans les années vingt: Clarté (1921-1928) », Revue française de Science politique, 1967, 3, p. 484-519.

44.

Michel TREBITSCH, «La mystique révolutionnaire dans les avant-gardes des années 1920 », dans Révolte et Société, Paris, Actes du $4^{\mathrm{e}}$ colloque d'Histoire au présent, Sources, travaux historiques et Publications de la Sorbonne, t. 1, 1989, p. 260-267.

45.

Discours de Stockholm, 1986, op. cit.

46.

Un certain reste d'enthousiasme, retranscrit dans un entretien avec Madeleine Chapsal (L'Express, 5 avril 1962), tempère la désespérance du roman Le Palace paru au même moment (Paris, Minuit, 1962).

47.

Mireille CALLE-GRUBER, Claude Simon. Une vie à écrire, op.cit., p. 265.

48.

Cécile yaPAUdjIAN-LABAT, "L'humanisme en question chez Claude Simon », dans Paul Dirkx et Pascal Mougin (dir.), Claude Simon: Situations, op. cit, p. 103-116.

49.

Claude SIMON, Discours de Stockholm, op. cit., p. 889.

50.

«Débat. Le romancier et la politique : "Et si les écrivains révolutionnaires jouaient le rôle de la presse du cœur ?” demande Claude Simon », L’Express, 25 juillet 1963, p. 25-26.

51.

La Quinzaine littéraire, 27, 1-15 mai 1967, p. 12-13.

52.

Claude SIMON, «Le rôle amoral de la culture », intervention du 19 janvier 1988 lors de la réunion des Nobel à Paris, Libération 20 janvier 1988, rééd. dans Mireille CALLE-GRUBER, Claude Simon. L'inlassable réancrage du vécu, Éditions de la Différence, 2011, p. 91-97.

53.

Dominique VIART, chapitre «La question de l'engagement », dans Une mémoire inquiète. La Route des Flandres de Claude Simon, op. cit., p. 226-254.

54.

Claude SIMON, « Le rôle amoral de la culture », art. cité, p. 94.

55.

Philippe ROGER, "Les Géorgiques de Claude Simon ou la Révolution ranimée », dans La légende de la Révolution au XXe siècle, Colloque Cerisy, Paris, Cinémas/Flammarion, 1988, p. 182-189.

56.

Entretien avec Jacqueline Platier, Le Monde, 4 septembre 1981, p. 11 et 13

57. 
Francine DUGAST-PORTES, « Le spectre de l'ascendance... », art. cité.

58.

Les Géorgiques, dans CEuvres II, coll. La Pléiade, Paris, Gallimard, 2013, p. 926.

59.

Alexandre DAUGE-ROTH, « Autobiographie et biographies parentales dans L'Acacia », art. cité.

60.

Pascal Mougin, « La mésalliance parentale », dans Paul Dirkx et Pascal Mougin (dir.), Claude Simon :

Situations, op. cit, p. 41-52.

61.

Alastair DUNCAN, « Allées et venues familiales chez Claude Simon », dans Jean-Yves Laurichesse (dir.), Claude Simon: Allées et venues. Actes du colloque international de Perpignan (14 et 15 mars 2003), Cahiers de l'université de Perpignan, $n^{\circ} 34,2004$, Perpignan, Presses universitaires, p. 171-181.

62.

Le Dictionnaire. Littérature française contemporaine, Jérôme Garcin (dir.), Paris, François Bourin, 1988, p. 401, notice biographique rédigée par Claude Simon lui-même.

63.

Claude SIMON, « Lieu », L’Humanité, 9 décembre 1977, p 2.

64.

Claude SIMON, Le Tricheur, Paris, Le Sagittaire, 1945, p. 78.

65.

Gérard GENETTE, «Langage poétique, poétique du langage », dans Figures II, Paris, Seuil, 1969, p. 123-153.

66.

Claude SIMON, « Littérature : tradition et révolution », art. cité.

\section{RÉSUMÉS}

L'écriture de Claude Simon a souvent été associée aux limites, voire à l'impossibilité, de toute restitution de la réalité historique par l'intermédiaire du langage. Le roman Les Géorgiques (1981), fait à partir d'archives léguées par un ancêtre Conventionnel, n'énonce pas de conclusions sur la Révolution française. Tout au plus, un tel texte brise l'épopée; la révolution - quel que soit le contexte historique - est à l'état de ruine, elle n'est plus un projet. Cependant, le motif de la révolution se voit tout autant rechargé d'une résonance languissante, à l'état en quelque sorte poétique. L'écrivain fait face à une triple injonction : référentielle, généalogique, mémorielle. En ce sens, ce n'est pas tant Claude Simon qui est l'héritier d'une révolution dont il pourrait disposer, mais bien plus elle - ses conséquences, son aura, sa mémoire - qui hérite d'un écrivain par l'intermédiaire des Géorgiques.

Claude Simon's writing style has often been associated with the limits, or even the impossibility, of producing a realistic depiction of history through language. His novel Les Géorgiques (1981), based on archives he inherited from an ancestor who participated in the National Convention, do not offer conclusive statements about the French Revolution. At best, a text such as this one aims at breaking the frame of this saga; the revolution-in whatever historical context-is in tatters, it is not a project anymore. However, at the same time, the pattern of the revolution is suffused 
with a languid resonance, in a sort of poetic condition. The author has to face a triple obligation: referential, genealogical, memorial. As a consequence, it is not so much Claude Simon who is the heir of a revolution that he can use how he wants to, than it is the revolution itself-its consequences, its aura, its memory-that inherits a writer through Les Géorgiques.

INDEX

Mots-clés : Roman, Archives, Mémoire, Famille, Langage

Keywords : Novel, Archives, Memory, Family, Language

\section{AUTEUR}

LOUIS HINCKER

Centre d'Histoire « Espaces \& Cultures » Université Clermont-Auvergne 